\title{
OPEN Experimental demonstration of negative refraction with 3D locally resonant acoustic metafluids
}

\author{
Benoit Tallon ${ }^{1}$, Artem Kovalenko ${ }^{2}$, Olivier Poncelet ${ }^{1}$, Christophe Aristégui ${ }^{1}$, \\ Olivier Mondain-Monval ${ }^{2} \&$ Thomas Brunet ${ }^{1 \bowtie}$
}

Negative refraction of acoustic waves is demonstrated through underwater experiments conducted at ultrasonic frequencies on a 3D locally resonant acoustic metafluid made of soft porous siliconerubber micro-beads suspended in a yield-stress fluid. By measuring the refracted angle of the acoustic beam transmitted through this metafluid shaped as a prism, we determine the acoustic index to water according to Snell's law. These experimental data are then compared with an excellent agreement to calculations performed in the framework of Multiple Scattering Theory showing that the emergence of negative refraction depends on the volume fraction $\Phi$ of the resonant micro-beads. For diluted metafluid $(\Phi=3 \%)$, only positive refraction occurs whereas negative refraction is demonstrated over a broad frequency band with concentrated metafluid $(\Phi=17 \%)$.

Since the pioneering works reported by Liu et al. ${ }^{1}$, locally resonant acoustic metamaterials have been attracting great attention ${ }^{2}$. One of the challenging issues has been the achievement of acoustic metamaterials with a negative refractive index that offer new possibilities for acoustic imaging materials and for the control of sound at sub-wavelength scales ${ }^{3}$. In acoustics, the refractive index $n$ is proportional to $\sqrt{\rho / K}$, where $K$ and $\rho$ are the bulk modulus and the mass density of the material. Many works have been devoted to the study of double-negative metamaterials ${ }^{4-12}$ for which the two constitutive parameters $K$ and $\rho$ are simultaneously negative, leading thus to a negative index ${ }^{13}$. It is worth noting that such a double-negativity condition is not required for (real) dissipative metamaterials to get a negative index ${ }^{14}$. When they are non-negligible, losses may play an important role in the effective acoustic properties of the metamaterials in such a way that the real part of the acoustic index can be negative for single-negative metamaterial as demonstrated in underwater ultrasonic experiments ${ }^{15}$, and in air at audible frequencies ${ }^{16}$. Although the latter work reported on the experimental observation of negative refraction effects within a $2 \mathrm{D}$ acoustic superlens, negative refraction has never been observed with a $3 \mathrm{D}$ acoustic metamaterial up to now.

In that context, we proposed to use a "soft" approach, combining various soft-matter techniques, to achieve soft $3 \mathrm{D}$ acoustic metamaterials with negative index composed of resonant porous micro-beads randomly-dispersed in a yield-stress fluid ${ }^{17}$. By taking benefit from the strong low-frequency Mie-type (monopolar and dipolar) resonances of these 'ultra-slow' particles, single-band ${ }^{15}$ and dual-band ${ }^{18}$ negative refractive indices were experimentally demonstrated. The issue of the experimental observation of negative refraction was then raised for these water-based metamaterials ${ }^{19}$, since the energy attenuation might be significant in these metafluids due to the intrinsic absorption in the porous micro-beads, and to the strong resonant scattering by the particles.

In this paper, we report on negative refraction experiments with metafluids, composed of soft porous siliconerubber micro-beads, exhibiting a negative acoustic index at ultrasonic frequencies ${ }^{15}$. In these experiments, the metafluid is confined in a prism-shaped box with a small angle $\left(\theta_{\text {fluid }}=+2^{\circ}\right)$ and with a very thin plastic surface in order to be acoustically transparent. As shown in Fig. 1, this metafluid is directly deposited on a large ultrasonic immersion transducer operating in water over a broad ultrasonic frequency range (from 15 to $600 \mathrm{kHz}$ ). The large dimensions of this transmitter ensures the generation of quasi-pure plane waves propagating in the metafluid along the vertical $z$-axis, from the bottom to the top. Then, the transmitted acoustic beam refracted by the interface metafluid/water is scanned in the water tank by using a small ultrasonic probe. Two samples, composed of micro-beads with similar mean diameters $d$ of about $750 \mu \mathrm{m}$, are considered in this study with

${ }^{1}$ Univ. Bordeaux - CNRS - Bordeaux INP - ENSAM, I2M, 33405 Talence, France. ${ }^{2}$ Univ. Bordeaux - CNRS, CRPP, 33600 Pessac, France. ${ }^{\bowtie}$ email: thomas.brunet@u-bordeaux.fr 

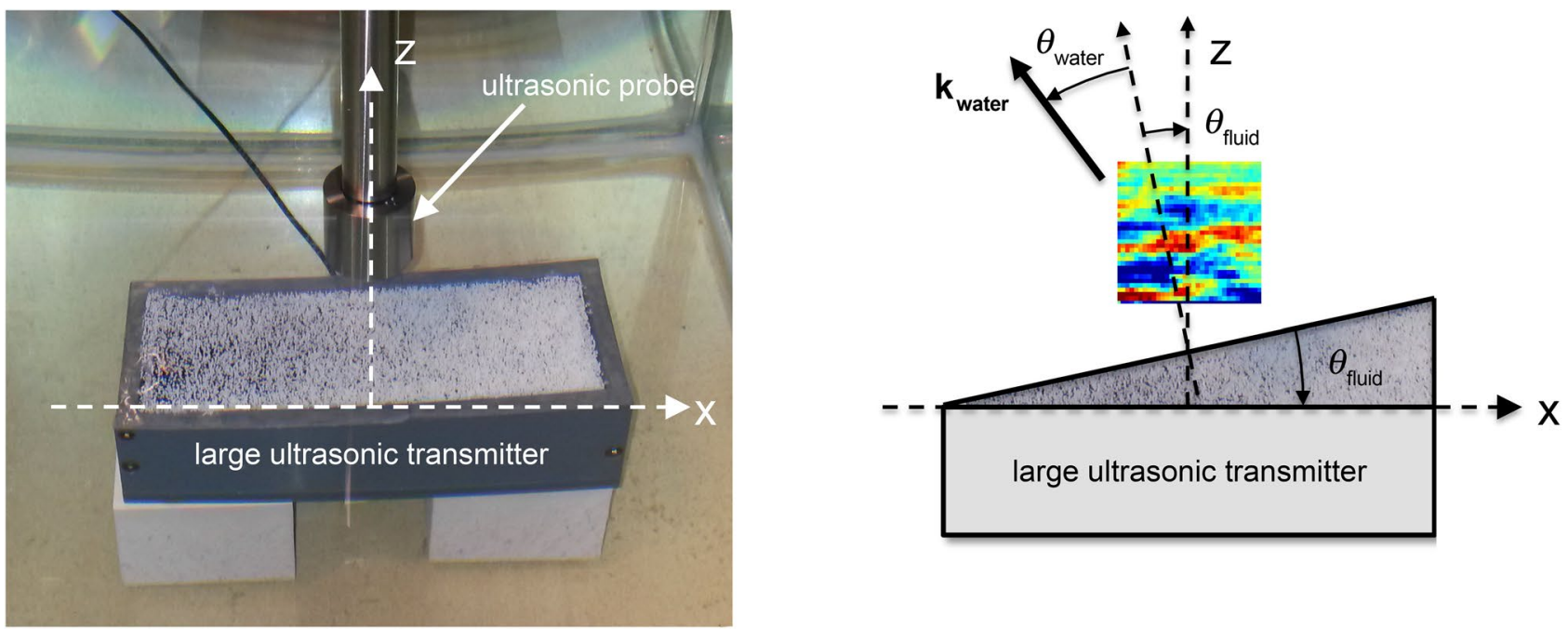

Figure 1. (left) Top view and (right) side view of the experimental setup. The metafluid is confined in a prismshaped box, with an angle $\theta_{\text {fluid }}=+2^{\circ}$, that is deposited directly on a large broad-band ultrasonic immersion transducer $(150 \mathrm{~mm} \times 40 \mathrm{~mm})$. The acoustic waves propagate from the bottom to the top of the water tank, along the vertical $z$-axis. The acoustic field refracted from the prism is scanned in the $x-z$ plane $(33 \mathrm{~mm} \times 33$ $\mathrm{mm}$ ) with a small ultrasonic probe.

two different volume fractions $\Phi$, referred to as the diluted metafluid $(\Phi=3 \%)$ and concentrated metafluid $(\Phi=17 \%)$. The acoustic properties of the micro-beads are given in a previous work ${ }^{20}$. One of the particular features of these porous particles is their very low longitudinal phase velocity $c_{L}\left(=120 \mathrm{~m} \mathrm{~s}^{-1}\right)$ that is due to the softness of the silicone-rubber material ${ }^{21}$.

\section{Results}

When excited with a short electrical pulse, the large ultrasonic transducer generates a short broad-band acoustic pulse propagating in the confined fluid along the $z$-axis only. At the interface between the confined fluid and water, the acoustic pulse is then refracted in the surrounding water with an angle of refraction $\theta_{\text {water }}$ shown in Fig. 1 . By measuring $\theta_{\text {water }}$, the acoustic index $n_{\text {fluid }}$ of the fluid confined in the prism can be easily deduced from the Snell's law as following:

$$
n_{\text {fluid }}=n_{\text {water }} \frac{\sin \left(\theta_{\text {water }}\right)}{\sin \left(\theta_{\text {fluid }}\right)}
$$

with $n_{\text {water }}=1$ since the acoustic index $n\left(=c_{0} / c\right)$ of a material with the phase velocity $c$ is usually defined relatively to water $\left(c_{0}=c_{\text {water }}\right)$ for underwater acoustics. In these experiments, the acoustic index $n_{\text {fluid }}$ cannot be directly retrieved from the refracted transmitted temporal signals measured in the $x-z$ plane as depicted in Fig. 1, since a short broad-band acoustic pulse has been used in these pulsed ultrasonic experiments. In a such broad frequency range (from $15 \mathrm{kHz}$ to $600 \mathrm{kHz}$ ), the acoustic index of a concentrated metafluid is expected to exhibit strong variations ranging from high positive values to negative ones ${ }^{15}$. Due to potential strong dispersion effects, we focus here on a harmonic spectral analysis of the refracted beams by performing Fourier transforms over time and space. First, we performed time-domain Fourier transforms of all the signals acquired at each position over a 2D spatial grid (Fig. 2a) in order to obtain the spatial-field at each frequency component (Fig. 2b). Then a $2 \mathrm{D}$ spatial Fourier transform is applied at each frequency for getting the wavenumber spectrum of the harmonic beam (Fig. 2c). Since the beams refracted in water are quasi-plane waves, it is straightforward to extract accurately their direction of propagation from the (real-valued) spatial Fourier components $k_{\mathrm{x}}^{\mathrm{r}}$ and $k_{\mathrm{z}}^{\mathrm{r}}$ at the peak amplitude of the wavenumber spectrum.

From the acoustic field map shown at a given time in Fig. 2a, we can get the corresponding map of the angular phase for each frequency component of the refracted beam. As an example, Fig. $2 b$ shows the angular phase of the refracted beam at $f=175 \mathrm{kHz}$ revealing an angle of refraction of a few degrees from the initially vertical propagation direction. Such a deviation can be estimated by measuring the tilted angle of these refracted wavefronts in the $x-z$ plane but this direct spatial measurement may suffer from fluctuations observed on the wavefronts. As an alternative, we performed 2D Fourier transforms of the scanned field patterns at different frequencies to infer the $k_{\mathrm{x}}^{\mathrm{r}}$ and $k_{\mathrm{z}}^{\mathrm{r}}$ coordinates of the wave vector $\mathbf{k}_{\text {water }}$ in the $k_{\mathrm{x}}-k_{\mathrm{z}}$ plane. The values of these two components are given by the location of the maximum spot intensity shown in Fig. $2 \mathrm{c}$. Then, the angle of refraction $\theta_{\text {water }}$ can be easily deduced for each frequency as following:

$$
\theta_{\text {water }}=\arctan \left(\frac{k_{\mathrm{x}}^{\mathrm{r}}}{k_{\mathrm{z}}^{\mathrm{r}}}\right)+\theta_{\text {fluid }}
$$



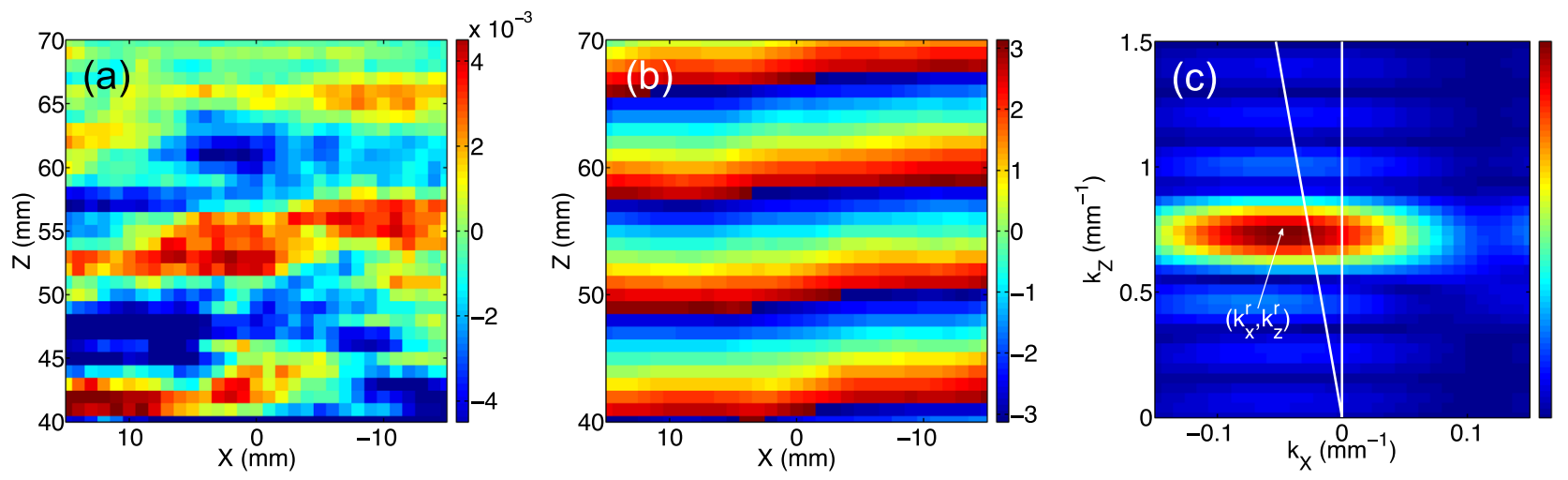

Figure 2. (a) Snapshot of the measured pressure field refracted from the prism filled with concentrated metafluid $(\Phi=17 \%)$. (b) Corresponding angular phase, shown here at $175 \mathrm{kHz}$, obtained from the timedomain Fourier transforms performed for each position over the square grid shown in (a). (c) 2D spatial Fourier transform of the scanned field pattern at $175 \mathrm{kHz}$ for the measurement of the (real-valued) spatial Fourier components $k_{\mathrm{x}}^{\mathrm{r}}$ and $k_{\mathrm{z}}^{\mathrm{r}}$ of the refracted beam in water.

In these experiments, the coordinate $k_{\mathrm{z}}^{\mathrm{r}}$ is necessarily positive since the acoustic waves propagate from the bottom to the top of water tank along the $z$-axis in any case. However, the coordinate $k_{\mathrm{x}}^{\mathrm{r}}$ may be either positive or negative depending on the direction of the refracted beam. The acoustic index $n_{\text {fluid }}$ of the fluid confined in the prism can be deduced from the values of the angle $\theta_{\text {water }}$ by using Eq. (1). When the prism is filled with water (Fig. 3, left), the spot goes along the vertical $k_{z}$-axis for which $k_{\mathrm{x}}^{\mathrm{r}}=0$ (white vertical line) as the frequency increases leading to $\theta_{\text {water }}=\theta_{\text {fluid }}$ according to Eq. (2). Therefore, no refraction occurs in that case which may be expected since the material is the same (water) on both sides of the interface. Note that if the spot had gone along the white titled line shown in Fig. 3, for which $\arctan \left(k_{\mathrm{x}}^{\mathrm{r}} / k_{\mathrm{z}}^{\mathrm{r}}\right)=-\theta_{\text {fluid, }}$, this would lead to $\theta_{\text {water }}=0$, corresponding thus to a zero-index fluid confined in the prism.

When the prism is filled with the diluted metafluid $(\Phi=3 \%, d=750 \mu \mathrm{m}$ with a size dispersion of $30 \%)$, the spot oscillates around the white vertical axis as the frequency increases (Fig. 3, center). The values of $n_{\text {fluid }}$ extracted from Eqs. (1) and (2) show that the acoustic index of this diluted metafluid varies from high values at low frequencies ( $n_{\text {fluid }}=+4$ at $40 \mathrm{kHz}$ ) to low values at intermediate frequencies $\left(n_{\text {fluid }}=+0.5\right.$ around $130 \mathrm{kHz}$ ) before getting closer to +1 at high frequencies as shown in Fig. 4 . This strong dispersion is due to the micro-bead resonances that occur around $150 \mathrm{kHz}$ for that size of particles. Far away from these low-frequency acoustic resonances, the acoustic index of the metafluid is similar to that of the aqueous matrix ${ }^{15}$.

We also produced a concentrated metafluid $(\Phi=17 \%, d=700 \mu \mathrm{m}$ with a size dispersion of $10 \%)$ in which negative refraction occurs for a certain range of frequencies. Actually, Fig. 3 (right) shows that the spot can go beyond the white tilted axis (corresponding to $n_{\text {fluid }}=0$ ) as observed at $160 \mathrm{kHz}$. The experimental results are not shown at $40 \mathrm{kHz}$ because of the very high attenuation that is due the strong (monopolar) low-frequency resonances of the micro-beads ${ }^{15}$. But the acoustic index is shown to be negative over a broad frequency range as shown in Fig. 4. Note that this 'negative band' is slightly shifted to higher frequencies compared to the diluted metafluid because of the smaller size of the micro-beads of this concentrated metafluid ${ }^{22}$.

Finally, we compared our acoustical measurements to theoretical predictions produced through multiplescattering modeling, revealing good qualitative agreement as shown in Fig. 4. The theoretical acoustic index of the metafluids $(\Phi=3 \%$ and $17 \%$ ) are obtained from their effective wavenumbers calculated using the Waterman-Truell formula ${ }^{23}$. The values of the material parameters for the soft porous silicone rubber that we used for these calculations, were $c_{L}=120 \mathrm{~m} \mathrm{~s}^{-1}, \alpha_{L}=20 \mathrm{~Np} \mathrm{~m}^{-1} \mathrm{MHz}^{-2}$ (the phase velocity and attenuation coefficient for longitudinal waves), $c_{T}=40 \mathrm{~m} \mathrm{~s}^{-1}, \alpha_{T}=200 \mathrm{~Np} \mathrm{~m}^{-1} \mathrm{MHz}^{-2}$ (the phase velocity and attenuation coefficient for shear waves) and $\rho_{1}=760 \mathrm{~kg} \mathrm{~m}^{-3}$. The water-based gel matrix has the same properties as water $\left(\rho_{0}=1000\right.$ $\mathrm{kg} \mathrm{m}^{-3}, c_{0}=1490 \mathrm{~m} \mathrm{~s}^{-1}$ ) since this host Bingham fluid is essentially made of water and very small amounts of polymer (Carbopol) to prevent the creaming of the porous micro-beads.

\section{Conclusion}

In summary, we have reported an experimental demonstration of negative refraction in a $3 \mathrm{D}$ acoustic metamaterial. The experiments have been conducted at ultrasonic frequencies with a locally resonant metafluid composed of soft porous silicone-rubber micro-beads whose concentration must be high enough so that refraction be negative. However, the achievement of acoustic devices based on negative refraction such as perfect lenses envisioned by Pendry ${ }^{24}$ seems unattainable with our metafluids because of their large attenuation that is mainly due to the strong resonant scattering ${ }^{15}$. Alternatively to bulky and lossy $3 \mathrm{D}$ metamaterials, acoustic metasurfaces ${ }^{25}$ might be much more appropriate to manipulate acoustic wavefronts as recently demonstrated with soft gradient-index metasurfaces $^{26}$. 

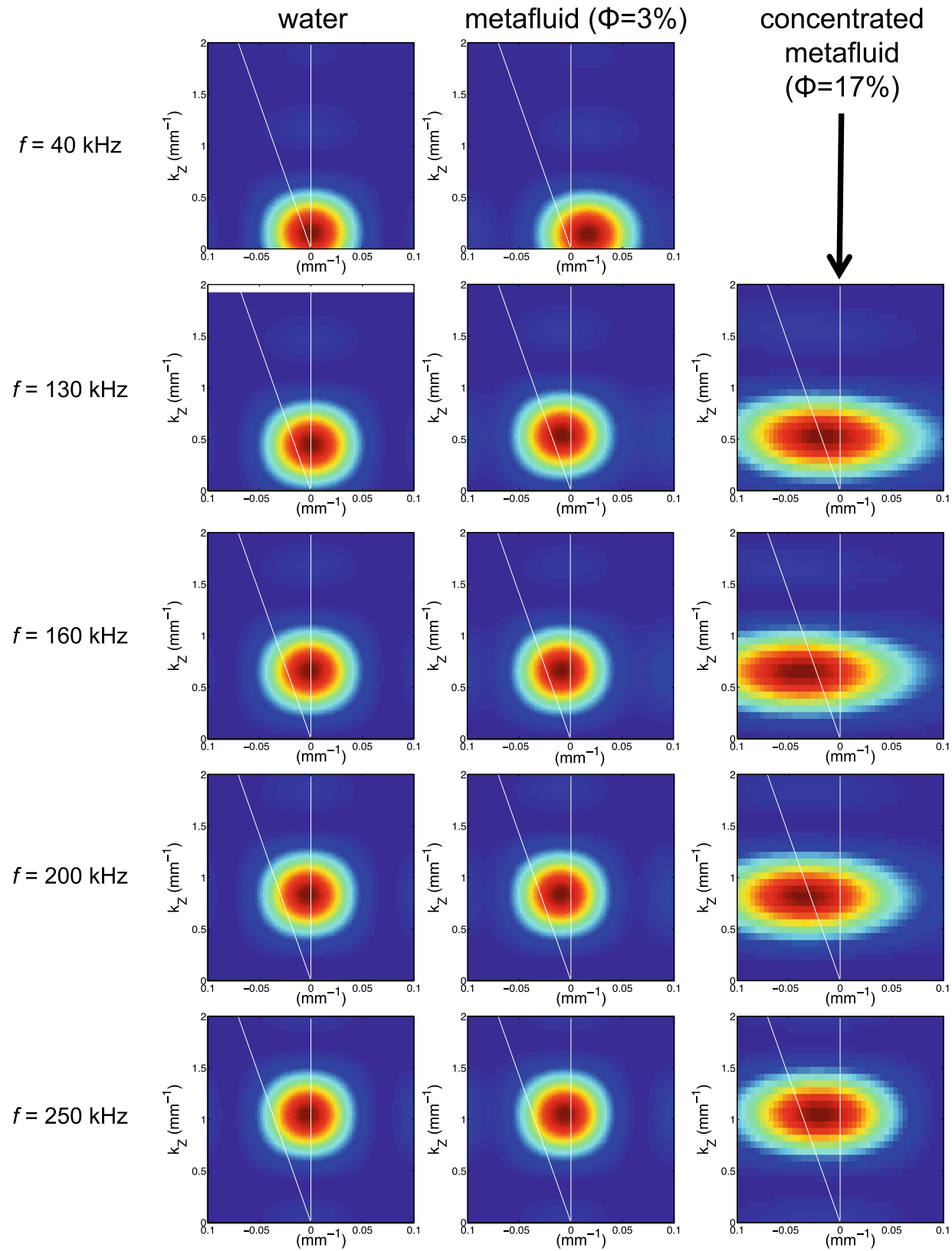

Figure 3. 2D Fourier transforms of the scanned field pattern performed at different frequencies: 40, 130, 160, $200,250 \mathrm{kHz}$ (from the top to bottom). The prism is filled with either water (left), diluted metafluid (center) or concentrated metafluid (right). On all maps, the white vertical axis $\left(k_{\mathrm{x}}=0\right)$ corresponds to the case in which $\theta_{\text {water }}=\theta_{\text {fluid }}$ meaning that $n_{\text {fluid }}=+1$, whereas the tilted white axis $\left(\arctan \left(k_{\mathrm{x}} / k_{\mathrm{z}}\right)=-\theta_{\text {fluid }}\right)$ corresponds to the case in which $\theta_{\text {water }}=0$ meaning that $n_{\text {fluid }}=0$. The Fourier transforms imply a plane wave in the positive $z$ direction.

\section{Methods}

In this study, we used a large ultrasonic transducer $(150 \mathrm{~mm} \times 40 \mathrm{~mm})$, with a central frequency of $150 \mathrm{kHz}$, to ensure the generation of quasi-pure plane waves in the water tank (propagating along the vertical axis from the bottom to the top of the water tank). This broad-band transducer was excited with a short electrical pulse generated by a pulser/receiver (Olympus, 5077PR) that was also used to amplify the electric signal recorded by the 1-inch-diameter receiving transducer (Olympus V301) before its acquisition on a computer via a waveform digitizer card (AlazarTech, ATS460). The angle of the prism has been chosen here as low as possible $\left(\theta_{\text {fluid }}=+2^{\circ}\right)$ in order to guarantee that the refracted-beam amplitude does not vary too much along the $\mathrm{x}$-axis in spite of the slightly increasing depth of the prism. For the experiments conducted with the prism filled with water (Fig. 3, left) or with the diluted metafluid (Fig. 3, centre), the width of the scan area along the $\mathrm{x}$-axis was $60 \mathrm{~mm}$. For the concentrated metafluid (Fig. 3, right), this width was reduced to $30 \mathrm{~mm}$ inducing a slight spread of the Fourier Transform distribution along the $k_{x}$-axis. The acoustical fields refracted in water were scanned on grids with a 


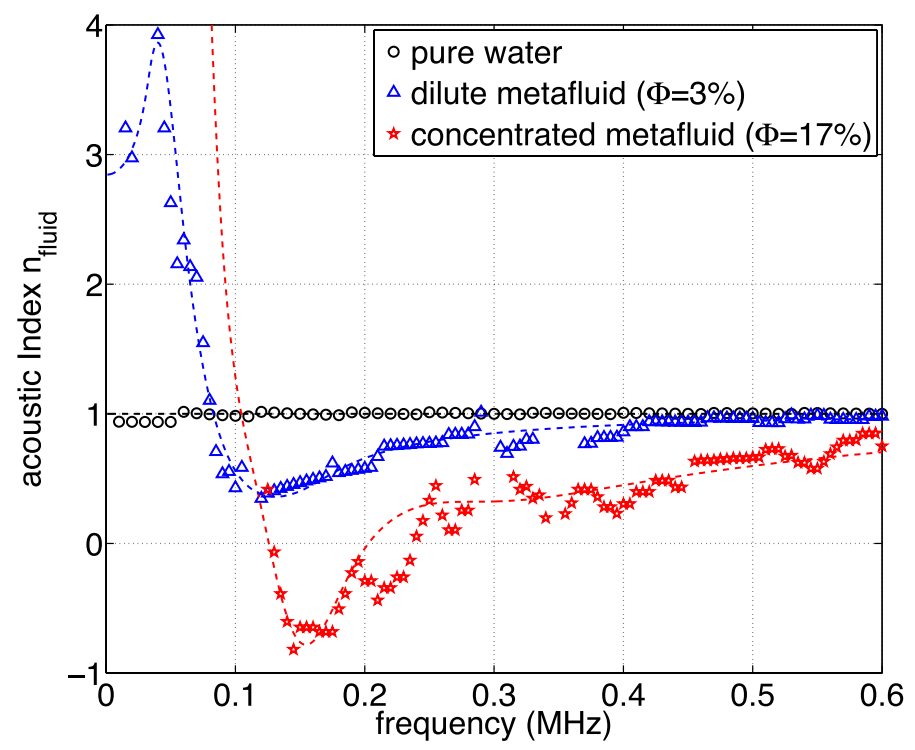

Figure 4. Acoustic index $n_{\text {fluid }}$ for different fluids confined in the prism-shaped box and extracted as a function of frequency from the $k$-maps. Dashed lines refer to calculations performed in the framework of multiple scattering theory (see the text).

step of $1 \mathrm{~mm}$ that is 3 times smaller than the acoustic wavelength in water at $500 \mathrm{kHz}(=3 \mathrm{~mm})$. Note that we also used zero-padding techniques before doing Fast Fourier Transforms in space to improve the resolution in the $k$-maps shown in Fig. 3.

Received: 28 February 2020; Accepted: 21 January 2021

Published online: 25 February 2021

\section{References}

1. Liu, Z. et al. Locally resonant sonic materials. Science 289, 1734-1736 (2000).

2. Ma, G. \& Sheng, P. Acoustic metamaterials: from local resonances to broad horizons. Sci. Adv. 2, e1501595. https://doi.org/10.1126/ sciadv.1501595 (2016).

3. Cummer, S. A., Christensen, J. \& Alù, A. Controlling sound with acoustic metamaterials. Nat. Rev. Mater. 1, 16001 EP (2016).

4. Li, J. \& Chan, C. T. Double-negative acoustic metamaterial. Phys. Rev. E 70, 055602 (2004).

5. Lee, S. H., Park, C. M., Seo, Y. M., Wang, Z. G. \& Kim, C. K. Composite acoustic medium with simultaneously negative density and modulus. Phys. Rev. Lett. 104, 054301 (2010).

6. Liang, Z., Willatzen, M., Li, J. \& Christensen, J. Tunable acoustic double negativity metamaterial. Sci. Rep. 2, 859 (2012).

7. Yang, M., Ma, G., Yang, Z. \& Sheng, P. Coupled membranes with doubly negative mass density and bulk modulus. Phys. Rev. Lett. 110, 134301. https://doi.org/10.1103/PhysRevLett.110.134301 (2013).

8. Maurya, S. K., Pandey, A., Shukla, S. \& Saxena, S. Double negativity in 3d space coiling metamaterials. Sci. Rep. 6, 33683. https:// doi.org/10.1038/srep33683 (2016).

9. Lanoy, M. et al. Acoustic double negativity induced by position correlations within a disordered set of monopolar resonators. Phys. Rev. B 96, 220201. https://doi.org/10.1103/PhysRevB.96.220201 (2017).

10. Zhou, Y., Fang, X., Li, D., Hao, T. \& Li, Y. Acoustic multiband double negativity from coupled single-negative resonators. Phys. Rev. Appl. 10, 044006. https://doi.org/10.1103/PhysRevApplied.10.044006 (2018).

11. Wang, W., Bonello, B., Djafari-Rouhani, B., Pennec, Y. \& Zhao, J. Double-negative pillared elastic metamaterial. Phys. Rev. Appl. 10, 064011. https://doi.org/10.1103/PhysRevApplied.10.064011 (2018).

12. Dong, H.-W., Zhao, S.-D., Wang, Y.-S., Cheng, L. \& Zhang, C. Robust 2d/3d multi-polar acoustic metamaterials with broadband double negativity. J. Mech. Phys. Solids 137, 103889. https://doi.org/10.1016/j.jmps.2020.103889 (2020).

13. Li, J., Fung, K. H., Liu, Z. Y., Sheng, P. \& Chan, C. T. Physics of Negative Refraction and Negative Index Materials (eds Krowne, C. M. \& Zong, Y.) (Springer, Berlin, 2007).

14. Brunet, T., Poncelet, O. \& Aristégui, C. Negative-index metamaterials: is double negativity a real issue for dissipative media?. EPJ Appl. Metamat. 2, 3. https://doi.org/10.1051/epjam/2015005 (2015).

15. Brunet, T. et al. Soft 3D acoustic metamaterial with negative index. Nat. Mater. 14, 384-388 (2015).

16. Kaina, N., Lemoult, F., Fink, M. \& Lerosey, G. Negative refractive index and acoustic superlens from multiple scattering in single negative metamaterials. Nature 525, 77-81 (2015).

17. Brunet, T., Leng, J. \& Mondain-Monval, O. Soft acoustic metamaterials. Science 342, 323-324. https://doi.org/10.1126/science.12417 27 (2013).

18. Raffy, S., Mascaro, B., Brunet, T., Mondain-Monval, O. \& Leng, J. A soft 3d acoustic metafluid with dual-band negative refractive index. Adv. Mater. 28, 1760-1764. https://doi.org/10.1002/adma.201503524 (2016).

19. Popa, B.-I. \& Cummer, S. A. Water-based metamaterials: negative refraction of sound. Nat. Mater. 14, 363-364 (2015).

20. Kovalenko, A., Zimny, K., Mascaro, B., Brunet, T. \& Mondain-Monval, O. Tailoring of the porous structure of soft emulsiontemplated polymer materials. Soft Matter 12, 5154-5163. https://doi.org/10.1039/C6SM00461J (2016).

21. Ba, A., Kovalenko, A., Aristégui, C., Mondain-Monval, O. \& Brunet, T. Soft porous silicone rubbers with ultra-low sound speeds in acoustic metamaterials. Sci. Rep. 7, 40106 EP (2017).

22. Brunet, T. et al. Sharp acoustic multipolar-resonances in highly monodisperse emulsions. Appl. Phys. Lett. 101, 011913. https:// doi.org/10.1063/1.4733615 (2012).

23. Waterman, P. C. \& Truell, R. Multiple scattering of waves. J. Math. Phys. 2, 512-537. https://doi.org/10.1063/1.1703737 (1961). 
24. Pendry, J. B. Negative refraction makes a perfect lens. Phys. Rev. Lett. 85, 3966-3969 (2000).

25. Assouar, B. et al. Acoustic metasurfaces. Nat. Rev. Mater. 3, 460-472. https://doi.org/10.1038/s41578-018-0061-4 (2018).

26. Jin, Y., Kumar, R., Poncelet, O., Mondain-Monval, O. \& Brunet, T. Flat acoustics with soft gradient-index metasurfaces. Nat. Commun. 10, 143. https://doi.org/10.1038/s41467-018-07990-5 (2019).

\section{Acknowledgements}

This work was partially funded and performed within the framework of the Labex AMADEUS ANR-10-LABEX0042-AMADEUS with the help of the French state Initiative d'Excellence IdEx ANR-10-IDEX-003-02 and project BRENNUS ANR-15-CE08-0024 (ANR and FRAE funds).

\section{Author contributions}

T.B. supervised the project, A.K. produced the soft porous silicone-rubber micro-beads to achieve the metafluids under the guidance of O.M.-M., B.T. and T.B. conducted the underwater experiments, B.T., T.B., C.A., and O.P analyzed the results. All authors reviewed the manuscript.

\section{Competing interests}

The authors declare no competing interests.

\section{Additional information}

Correspondence and requests for materials should be addressed to T.B.

Reprints and permissions information is available at www.nature.com/reprints.

Publisher's note Springer Nature remains neutral with regard to jurisdictional claims in published maps and institutional affiliations.

Open Access This article is licensed under a Creative Commons Attribution 4.0 International License, which permits use, sharing, adaptation, distribution and reproduction in any medium or format, as long as you give appropriate credit to the original author(s) and the source, provide a link to the Creative Commons licence, and indicate if changes were made. The images or other third party material in this article are included in the article's Creative Commons licence, unless indicated otherwise in a credit line to the material. If material is not included in the article's Creative Commons licence and your intended use is not permitted by statutory regulation or exceeds the permitted use, you will need to obtain permission directly from the copyright holder. To view a copy of this licence, visit http://creativecommons.org/licenses/by/4.0/.

(C) The Author(s) 2021 